\title{
Therapie der allergischen Rhinitis: Gefragt ist schnelle Symptomlinderung
}

— Für Patienten mit allergischer Rhinitis (AR) ist immer Hochsaison. Bereits im Januar startet die Saison mit den Haselnusspollen, geht im März weiter mit Birkenpollen und dauert dank Ambrosia bis in den Herbst. Im Winter leiden Betroffene vor allem in geschlossenen Räumen unter Tierhaaren und Schimmelpilzen. Der Wunsch nach einer wirksamen Soforthilfe ist groß. Die Ergebnisse einer Umfrage zeigen nun, dass die behandelnden Ärzte ganz ähnliche Wünsche an ein Medikament zur Behandlung der AR haben wie ihre Patienten. In der ersten Jahreshälfte 2016 wurden Allgemeinmediziner $(n=110)$ zur Behandlung der allergischen Rhinitis und des allergischen Asthmas befragt. Dabei ging es unter anderem darum, welche Eigenschaften das ideale Medikament haben sollte und welche Therapieformen sie bevorzugt empfehlen. Befragt nach der bevorzugten Darreichungsform kristallisierten sich bei den Teilnehmern der Umfrage $(n=110)$ zwei Spitzenreiter heraus: $91 \%$ der Befragten empfehlen vor allem Nasensprays, 75\% empfehlen orale Antihistaminika. Bevorzugt werden von den Allgemeinmedizinern also Therapien, die kurzfristig und einfach anzuwenden sind, da sie die Therapie-Adhärenz der Patienten erhöhen.
Die Umfrage unter den Ärzten ergab auch, dass die wichtigste Eigenschaft eines Medikaments zur Behandlung der AR ein schneller Wirkeintritt ist. Dahinter rangieren die Verbesserung der nasalen und okularen Symptome, gute Verträglichkeit und eine langanhaltende Wirkung. Dies spiegelt auch die Bedürfnisse der betroffenen Patienten wider, die sich häufig eine rasche und vollständige Symptomlinderung wünschen, die oft mit den aktuell verfügbaren Monotherapieoptionen nicht erreicht werden kann. Eines der Probleme dabei ist, dass der Patient das Vertrauen in die Therapie verlieren kann, wenn die ersten Therapieversuche nicht anschlagen: Studien zeigen, dass zwei von drei Betroffenen mit ihrer Medikation unzufrieden sind.

Dymista $^{\circledR}$ Nasenspray bietet mit seiner Fixkombination aus Azelastinhydrochlorid und Flucticasonpropionat genau die von Ärzten und Patienten gewünschten Eigenschaften: $65 \%$ der Teilnehmer an der Umfrage sehen in der höheren Wirksamkeit im Vergleich zu Monosubstanzen einen klaren Vorteil der Wirkstoffkombination. Die deutliche Verbesserung der nasalen und der okularen Symptome sehen $57 \%$ der Befragten als wichtigen Vorteil, den schnellen Wirkeintritt heben

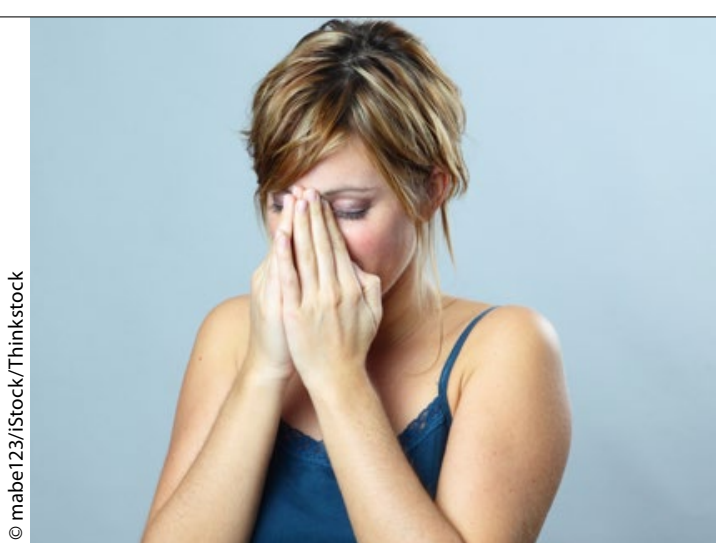

Patienten mit allergischer Rhinitis wünschen sich wirksame Soforthilfe.

$45 \%$ hervor. Durch die einzigartige Galenik wird eine einfache medikamentöse Therapie bei mittelschwerer bis schwerer saisonaler und perennialer AR ermöglicht, wenn eine Monotherapie (Antihistaminikum/Steroid) nicht als ausreichend erachtet wird. Ein weiterer Vorteil von Dymista ${ }^{\circledR}$ Nasenspray besteht im schnellen Wirkeintritt und der langanhaltenden Wirksamkeit: Bereits nach drei Tagen geben $50 \%$ der Patienten eine gute Symptomkontrolle an. Damit verbessert es auch mitten in der Hochsaison die Lebensqualität der Betroffenen erheblich. Die Umfrage zeigte auch, für welche Allergene Dymista ${ }^{\oplus}$ Nasenspray am häufigsten verordnet wird: $77 \%$ gaben an, es bevorzugt bei Pollenallergien einzusetzen, $29 \%$ verschreiben es auch bei Hausstauballergien.

red

Nach Informationen von MEDA Pharma

\section{Neues Wireless-Gerät für Träger von Cochlea- Implantaten}

- Cochlear erweitert sein Portfolio an Wireless-Geräten um eine weitere Lösung. Das Cochlear ${ }^{\mathrm{TM}}$ Wireless Minimikrofon $2+$ bietet Trägern eines Cochlear $^{\mathrm{TM}}$ Nucleus $^{\circledR} 6$ Soundprozessors kabellose Freiheit und zusätzliche Unterstützung beim Hören in akustisch anspruchsvollen Situationen. $\mathrm{Ob}$ in Schule, Beruf oder Freizeit - das neue Minimikrofon 2+ überträgt Sprache und Audiosignale komfortabel und zuverlässig über eine Entfernung von bis zu 25 Metern direkt zum Soundprozessor. Darüber hinaus kann das Wireless-Gerät auch als Tischmikrofon fungieren. Es verfügt über eine Telefonspulen- und eine FM-Funktion. Und es kann über einen Audioeingang plug-andplay mit TV, Musikanlage und Computer gekoppelt werden. Der Ton wird dann direkt in die Soundprozessoren übertragen. „Mit dem Minimikrofon 2+ können Träger von Nucleus 6 das Verhältnis von Signal und Störgeräusch deutlich optimieren", so Frederec Lau, Marketingleiter von Cochlear Deutschland. „Unser neuestes Wireless-Gerät überträgt auf Basis der 2,4-GHz-Funktechnologie die Stimme eines Gesprächspartners; dafür sind keine zusätzlichen Geräte erforderlich. Das Signal wird stark, klar und stabil direkt im Soundprozessor empfangen. Vor allem in Situationen, in denen die Entfernung zwischen den Gesprächspartnern, Hintergrundgeräusche oder schlechte Raumakustik das Hören erschweren, verbessert sich das Sprachverstehen deutlich. Zu- gleich kann sich der Nutzer frei und ohne jede Einschränkung bewegen."

Die Handhabung des Minimikrofons 2+ ist einfach. Die Synchronisation, die an Mikrofon und Soundprozessor oder alternativ mittels Cochlear Anpasssoftware erfolgt, bleibt dauerhaft erhalten und muss nicht wiederholt werden. Vor jeder Nutzung wird das Mikrofon eingeschaltet und die Wireless-Übertragung am Soundprozessor aktiviert. Die Regelung der Lautstärke des Minimikrofons erfolgt über Tasten. Sie ist im Bereich von $-24 \mathrm{~dB}$ bis $+12 \mathrm{~dB}$ möglich. Ein Fall-Sensor garantiert, dass sich das Gerät beim Fall aus einer Höhe von über $75 \mathrm{~cm}$ für kurze Zeit stumm schaltet. Betrieben wird das Mikrofon über ein Akku; dessen Laufzeit liegt je nach verwendetem Modus bei sieben bis elf Stunden.

red

Nach Informationen von Cochlear 